\title{
Largest component and node fault tolerance for grids
}

\author{
Jakub Przybyło* Andrzej Żak \\ Faculty of Applied Mathematics \\ AGH University of Science and Technology \\ Kraków, Poland \\ \{jakubprz, zakandrz\}@agh.edu.pl
}

Submitted: Dec 10, 2018; Accepted: Jan 20, 2021; Published: Feb 26, 2021

(C) The authors. Released under the CC BY-ND license (International 4.0).

\begin{abstract}
A graph $G$ is called $t$-node fault tolerant with respect to $H$ if $G$ still contains a subgraph isomorphic to $H$ after removing any $t$ of its vertices. The least value of $|E(G)|-|E(H)|$ among all such graphs $G$ is denoted by $\Delta(t, H)$. We study fault tolerance with respect to some natural architectures of a computer network, i.e. the $d$-dimensional toroidal grids and the hypercubes. We provide the first nontrivial lower bounds for $\Delta(1, H)$ in these cases. For this aim we establish a general connection between the notion of fault tolerance and the size of a largest component of a graph. In particular, we give for all values of $k$ (and $n$ ) a lower bound on the order of the largest component of any graph obtained from $C_{n} \square C_{n}$ via removal of $k$ of its vertices, which is in general optimal.
\end{abstract}

Mathematics Subject Classifications: 05C70, 05C78

\section{Introduction}

A graph $G$ is called $t$-node fault tolerant with respect to $H$ ( $t$-FT $(H)$ for short) if $G-S$ contains $H$ as a subgraph for every $S \subset V(G)$ with $|S| \leqslant t$. This problem was introduced by Hayes [9] in 1976 as a graph theoretic model of computer or communication networks operating correctly in the presence of faults. Different measures of efficiency of faulttolerant graphs have been studied so far. Hayes [9] and Ajtai et al. [1] considered $t$ $\mathrm{FT}(H)$ graphs that have exactly $t$ spare nodes and as small as possible number of edges. Alon and Chung [2], Zhang [14], Ueno and Yamada [12], alowed $O(t)$ spare nodes and focused on minimizing the maximum degree to maintain a scalability of a network. In this paper we study another variant introduced by Ueno et al. [11], and independently

*This work was partially supported by the Faculty of Applied Mathematics AGH UST statutory tasks within subsidy of Ministry of Science and Higher Education. 
by Dudek et al. in [7]. In this variant one is interested in $t$-FT $(H)$ graphs having as few as possible edges, disregarding the number of vertices (note that Erdös, Graham and Szemerédi [8] considered already the analogue of this variant for dipaths of order exactly $t$ ). Let $\Delta(G, H)=|E(G)|-|E(H)|$ and define

$$
\Delta(t, H)=\min \{\Delta(G, H): G \text { is } t-\mathrm{FT}(H)\} .
$$

Ueno et al. [11] determined the growth order $\Delta(t, H)=\Theta(\sqrt{|H|})$, where $t$ is fixed and $|H|$ tends to infinity, for some special families of graphs. They conjectured [11, pp. 571] that there exists a general lower bound for connected graphs $H$ and $t \geqslant 2$ where $c$ is a universal constant:

$$
\Delta(t, H) \geqslant c \sqrt{t|H|}+f(t)
$$

The second author proved the conjecture in the case of regular graphs [15, Theorem 1]. Theorem 1 ([15]). Let $H$ be an $r$-regular graph of connectivity $\kappa$, and let $t \geqslant 1$. If $r(|H|+1-\kappa) \geqslant 2 \sqrt{r \kappa t|H|}+t(1+r-r \kappa)$ then

$$
\Delta(t, H) \geqslant \sqrt{r \kappa t|H|}-\frac{t(r \kappa-r-1)}{2} .
$$

For $t=1$, the above bound is close to optimal for many families of graphs, however, for some typical widely studied architectures of computer networks, the growth order is suspected to be significantly bigger. Ueno et al. [11, Sections 6-7] considered (among other graphs) the toroidal grids $C_{n} \square C_{n}$ and the hypercubes $Q_{n}$. They proved that $\Delta(1, H)=$ $O\left(|H|^{3 / 4}\right)$ in the case when $H=C_{n} \square C_{n}$, see [11, Theorem 11], and conjectured that this is the right growth rate. However, they did not provide any lower bound neither for the toroidal grid nor for the hypercube, which is essentially bigger than (1). In this paper we improve the known lower bounds significantly, by establishing the following general relation between the notion of 1-FT $(H)$ graphs and the size of a largest component of a graph obtained by removing a given number of its vertices. Let $m(G)$ denote the number of vertices in a largest component of $G$ and let

$$
m(G, k)=\min \{m(G-S): S \subset V(G),|S|=k\} .
$$

Theorem 2. Let $H$ be an $r$-regular graph, $r \geqslant 1$. Then

$$
\Delta(1, H) \geqslant \frac{1}{2} \min _{0 \leqslant k \leqslant|V(H)|}\{r m(H, k)+k\} .
$$

In particular, we may derive many new bounds for $\Delta(1, H)$ from known results on the integrity of graphs, c.f. [3]. The integrity $I(H)$ of a graph $H$ is defined as follows

$$
I(H)=\min \{m(H-S)+|S|: S \subset V(H)\} .
$$

Thus we immediately obtain the following corollary. 
Corollary 3. Let $H$ be an $r$-regular graph, $r \geqslant 1$. Then

$$
\Delta(1, H) \geqslant \frac{1}{2} I(H)
$$

We give special attention to the toroidal grids since we believe that our estimation on the parameter $m\left(C_{n} \square C_{n}, k\right)$ may be of independent interest. Thus, although much less is sufficient within the mentioned application, we nevertheless provide a general result for the entire spectrum of potential values of $k$.

Theorem 4 . For any positive integers $n \geqslant 3$ and $k$

$$
m\left(C_{n} \square C_{n}, k\right) \geqslant \begin{cases}n^{2}-\frac{(k+2)^{2}}{8}-\frac{1}{2} & \text { for } 1 \leqslant k \leqslant 2 n-3 \\ \frac{(n-1)^{2}}{2}+\frac{1}{2}, & \text { for } k=2 n-2 \\ \frac{\left(n^{2}-k\right)}{k^{2}} \cdot\left(n^{2}-k+n \sqrt{n^{2}-2 k}\right), & \text { for } 2 n-1 \leqslant k<\frac{n^{2}}{2} \\ 1, & \text { for } \frac{n^{2}}{2} \leqslant k \leqslant n^{2}-1 .\end{cases}
$$

We moreover show that all bounds in Theorem 4 above are in general optimal, i.e. these are tight in infinitely many cases. Note that whenever $n \ll k \ll n^{2}$, the lower bound in Theorem 4 is roughly $2 n^{4} / k^{2}$.

As an application of Theorem 2, Theorem 4 and Corollary 3 we obtain the following lower bounds on $\Delta(1, H)$. We say that a graph $G$ on $n$ vertices is $(\alpha, \beta)$-expander if for any set $A \subset V(G)$ such that $|A| \leqslant \alpha n$, its external neighborhood satisfies $|N(A)| \geqslant \beta|A|$. Furthermore, denote

$$
C_{n}^{d}=\underbrace{C_{n} \square \cdots \square C_{n}}_{d} .
$$

\section{Theorem 5.}

1. There exists $n_{0}$ such that for every $n>n_{0}$

$$
\Delta\left(1, C_{n} \square C_{n}\right) \geqslant 3 n^{4 / 3} .
$$

2. There exists a constant $c_{d}$ depending only on $d$ such that

$$
\Delta\left(1, C_{n}^{d}\right) \geqslant c_{d} n^{d^{2} /(d+1)} .
$$

3. There exists a constant $c>0$ such that

$$
\Delta\left(1, Q_{n}\right) \geqslant c \frac{2^{n}}{\sqrt{n}}
$$

4. Let $H$ be an $r$-regular $(\alpha, \beta)$-expander on $n$ vertices. Then

$$
\Delta(1, H) \geqslant \frac{n}{2} \min \left\{r \alpha, \frac{\beta}{r+\beta}\right\} .
$$


Note that the parameter $\Delta(1, H)$ in Theorem 5 is, respectively, of order $\Omega\left(|H|^{2 / 3}\right)$, $\Omega\left(|H|^{d /(d+1)}\right), \Omega(|H| / \sqrt{\log |H|}), \Omega(|H|)$, and thus exceeds (1) significantly.

In what follows by a graph we mean a simple graph without multiple edges and loops. Given a graph $G, V(G)$ denotes the vertex set of $G$ and $E(G)$ denotes the edge set of $G$. Furthermore, $|V(G)|$ is the order of $G$ and $|E(G)|$ is the size of $G$. By $N_{G}(x)$ we denote the set of vertices adjacent with $x$ in $G$. For a vertex set $X \subseteq V(G), G[X]$ denotes the subgraph of $G$ induced by $X$, while the set $N_{G}(X)$ denotes the external neighbourhood of $X$ in $G$, i.e.

$$
N_{G}(X)=\{y \in V(G) \backslash X: y \text { is adjacent with some } x \in X\} .
$$

Moreover, if $H$ is a subgraph of $G$, by the external neighbourhood of $H, N_{G}(H)$, we understand $N_{G}(V(H))$.

\section{Fault tolerance}

\section{$2.1 \quad$ Proof of Theorem 2}

We begin with the following obvious observation.

Observation 6. Let $H$ be a subgraph of $G$ and let $d_{G}(v)<\delta(H)$ for some $v \in V(G)$. Then $H$ is a subgraph of $G-v$.

Proof of Theorem 2. . Let $G$ be a 1-FT $(H)$ graph with the minimum number of edges. By Observation 6 , we may assume that $\delta(G) \geqslant r$. Let $K$ be the subset of $V(G)$ consisting of all vertices of $G$ with degree at least $r+1$, and set $k=|K|$. Let $C$ be a largest component (i.e. of largest order) of $G-K$. Since $H$ is a subgraph of $G,|C| \geqslant m(G, k) \geqslant m(H, k)$. We will show that $H$ is a subgraph of $G-V(C)$.

Let $v_{0}$ be any vertex of $C$ and set $G_{1}=G-v_{0}$. Since $G$ is $1-\mathrm{FT}(H), H$ is a subgraph of $G_{1}$. We furthermore, for $i=1, \ldots,|C|$ define $G_{i+1}:=G_{i}-v_{i}$ where $v_{i} \in V\left(G_{i}\right) \cap C$ with $d_{G_{i}}\left(v_{i}\right)<r$. To see that we can always find such $v_{i}$, recall that $d_{G}(v)=r$ for each $v \in C$ and $V\left(G_{i}\right)$ has at least one neighbor in $\left\{v_{0}, \ldots, v_{i-1}\right\}$ in $G$ (because $C$ is connected in $G$ ). By Observation $6, H$ is a subgraph of $G_{i}-v_{i}$ for every $i=1, \ldots,|C|$. In particular, $H$ is thus a subgraph of $G-V(C)$.

Therefore,

$$
|V(G)| \geqslant|V(H)|+|C| \geqslant|V(H)|+m(H, k)
$$

Hence,

$$
\begin{aligned}
|E(G)|-|E(H)| & \geqslant \frac{r(|V(G)|-k)+(r+1) k}{2}-\frac{r|V(H)|}{2} \\
& \geqslant \frac{r(|V(H)|+m(H, k)-k)+(r+1) k-r|V(H)|}{2} \\
& =\frac{r m(H, k)+k}{2}
\end{aligned}
$$




\subsection{Proof of Theorem 5}

In this section we complete the proof of Theorem 5 .

Proof of Theorem 5. We prove cases 1-4 separately.

1. Denote

$$
g(n, k):=4 m\left(C_{n} \square C_{n}, k\right)+k .
$$

By Theorem 2,

$$
\Delta\left(1, C_{n} \square C_{n}\right) \geqslant \frac{1}{2} \min _{0 \leqslant k \leqslant n^{2}} g(n, k) .
$$

In order to prove the statement, we will show that if $n$ is large enough, then for every integer $k \in\left[0, n^{2}\right]$,

$$
g(n, k) \geqslant 3 n^{4 / 3}
$$

This is obvious by the definition of $g(n, k)$ if $k \geqslant 3 n^{4 / 3}$, while it follows by Theorem 4 for $k \leqslant 2 n-2$. For the remaining values of $k$, by Theorem 4 we however have:

$$
g(n, k) \geqslant \frac{4 n^{4}}{k^{2}}+k
$$

(for $n$ large enough). Consider $f(x)=\frac{4 n^{4}}{x^{2}}+x, x \in\left[2 n-1,3 n^{4 / 3}\right]$. The derivative of $f(x)$ equals $f^{\prime}(x)=-8 \frac{n^{4}}{x^{3}}+1$. Clearly,

$$
f^{\prime}(x)>0 \Longleftrightarrow x^{3}>8 n^{4} .
$$

Hence, $f$ attains its minimum for $x_{0}=2 n^{4 / 3}$, and $f\left(x_{0}\right)=3 n^{4 / 3}$, and thus (2) follows.

2. We derive the proof from known results. Benke et al. [6] proved the following theorem.

Theorem 7 ([6], Thm. 1.6). There exist a constant $c_{d}>0$ depending only on $d$, such that

$$
I\left(P_{n}^{d}\right) \geqslant c_{d} n^{d^{2} /(d+1)} .
$$

Clearly, $I\left(C_{n}^{d}\right) \geqslant I\left(P_{n}^{d}\right)$. Hence, the proof follows by Corollary 3. (Note that the growth order for the bound in case 1 of Theorem 5 follows from case 2 , as well, however, with a much worse constant $c_{2}=0.00136$ (cf. [6], Thm. 1.5)).

3. So far the best known bounds on the integrity of a hypercube are due to Beineke et al. [5] (lower bound) and Balogh et al. [4] (upper bound).

Theorem $8([4,5])$. There exist constants $c, C>0$ such that

$$
c \frac{2^{n}}{\sqrt{n}} \leqslant I\left(Q_{n}\right) \leqslant C \frac{2^{n}}{\sqrt{n}} \sqrt{\log n} .
$$

Hence the proof of this case follows from Corollary 3, again. 
4. Let $S \subset V(G)$ with $|S|=k$. Let $A_{1}, \ldots, A_{l}$ be connected components of $G-S$. If there exists $i$ such that $\left|A_{i}\right|>\alpha n$ then, by Theorem $2, \Delta(1, H) \geqslant \frac{r \alpha n}{2}$. Otherwise, by the expanding property,

$$
r k=r|S| \geqslant \sum_{i=1}^{l}\left|N\left(A_{i}\right)\right| \geqslant \sum_{i=1}^{l} \beta\left|A_{i}\right|=\beta(n-k) .
$$

Hence $k \geqslant n \frac{\beta}{r+\beta}$ and the statement follows by Theorem 2, too. (a similar simple proof appeared in [13], where the integrity of cubic graphs was considered).

\section{Largest component in the toroidal grid}

\subsection{Preliminaries}

Let $G=C_{n} \square C_{n}$ or $G=P_{n} \square P_{n}$, where $V(G)=\{1,2, \ldots, n\} \times\{1,2, \ldots, n\}$, and $\{(i, j),(k, l)\} \in E(G)$ iff $i=k$ and $|j-l|=1$ (or $\{j, l\}=\{1, n\}$ in the case of $G=C_{n} \square C_{n}$ ) or $j=l$ and $|i-k|=1$ (or $\{i, k\}=\{1, n\}$ in the case of $G=C_{n} \square C_{n}$ ). If $(i, j),(k, l) \in V(G)$ then we say that the distance between $(i, j)$ and $(k, l)$ is

- 1 if $\{(i, j),(k, l)\} \in E(G)$

- $\sqrt{2}$ if $|i-k|=1$ (or $\{i, k\}=\{1, n\}$ in the case of $G=C_{n} \square C_{n}$ ) and $|j-l|=1$ (or $\{j, l\}=\{1, n\}$ in the case of $G=C_{n} \square C_{n}$ )

$\bullet \geqslant 2$ otherwise

In the same manner we define the distance in $G=P_{\infty} \square P_{\infty}$. In all these cases, given $S \subset V(G)$, by $G_{S}^{\prime}$ we denote the graph $\left(S, E_{S}^{\prime}\right)$ where $u v \in E_{S}^{\prime}$ if and only if the distance between $u$ and $v$ is less than or equal to $\sqrt{2}$ in $G$. Finally, by $G_{S}$ we denote the graph that is obtained from $G_{S}^{\prime}$ by removing both diagonals from each copy of $K_{4}$ in $G_{S}^{\prime}$, see an example on Figure 1. Clearly, $G_{S}$ has no crossing edges and so is embeddable on the plane or torus depending on which surface $G$ is embeddable. We first prove the following simple geometric fact.

Proposition 9 . Let $P$ be a planar polygon with all angles equal to $\pi / 2$ or $3 \pi / 2$. If the circumference of $P$ is less than or equal to $a$, then:

$$
|P| \leqslant\left(\frac{a}{4}\right)^{2}
$$

Proof. We may assume that $P$ is represented on a plane with all sides parallel to the axis of a coordinate system of the plane. Let $\left[x_{0}, x_{1}\right]$ be the projection of $P$ onto the $x$-axis, and $\left[y_{0}, y_{1}\right]$ be the projection of $P$ onto the $y$-axis. Then the rectangle $R:=\left[x_{0}, x_{1}\right] \times\left[y_{0}, y_{1}\right]$ contains $P$. Thus $|P| \leqslant|R|$. On the other hand, $c(R) \leqslant c(P) \leqslant a$, where by $c\left(P^{\prime}\right)$ we understand the circumference of any polygon $P^{\prime}$. To see this note that $\left(x_{0}, y^{\prime}\right),\left(x_{1}, y^{\prime \prime}\right)$, $\left(x^{\prime}, y_{0}\right),\left(x^{\prime \prime}, y_{1}\right)$ are nodes of $P$ for some $x^{\prime}, x^{\prime \prime}, y^{\prime}, y^{\prime \prime}$. Since all sides of $P$ are parallel to 

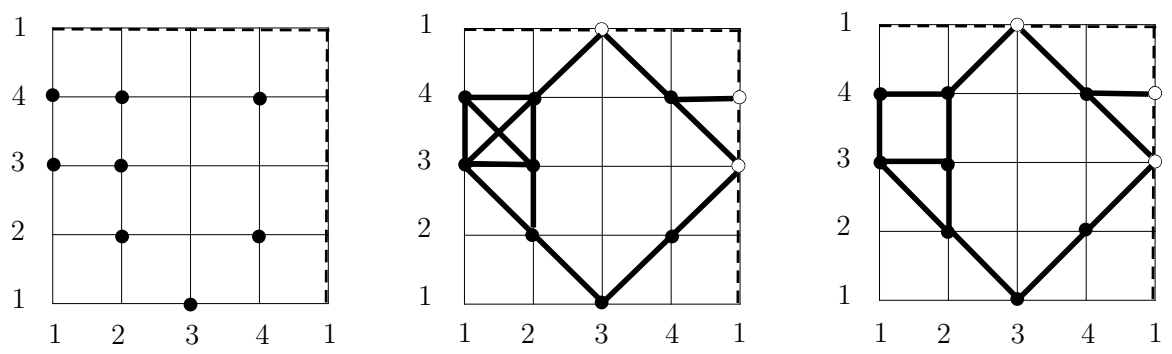

Figure 1: $S \subset C_{4} \square C_{4}$ (black dots), $G_{S}^{\prime}$ and $G_{S}$.

the axis, the polyline $\left(x_{0}, y^{\prime}\right) \rightarrow\left(x_{0}, y_{1}\right) \rightarrow\left(x^{\prime \prime}, y_{1}\right)$ (formed of two line segments joining $\left(x_{0}, y^{\prime}\right)$ with $\left(x_{0}, y_{1}\right)$ and $\left(x_{0}, y_{1}\right)$ with $\left.\left(x^{\prime \prime}, y_{1}\right)\right)$ has length less than or equal to the length of any polyline formed by sides of $P$ and joining $\left(x_{0}, y^{\prime}\right)$ with $\left(x^{\prime \prime}, y_{1}\right)$, and analogous statements hold for polylines $\left(x^{\prime \prime}, y_{1}\right) \rightarrow\left(x_{1}, y_{1}\right) \rightarrow\left(x_{1}, y^{\prime \prime}\right),\left(x_{1}, y^{\prime \prime}\right) \rightarrow\left(x_{1}, y_{0}\right) \rightarrow\left(x^{\prime}, y_{0}\right)$ and $\left(x^{\prime}, y_{0}\right) \rightarrow\left(x_{0}, y_{0}\right) \rightarrow\left(x_{0}, y^{\prime}\right)$. Moreover, the surface area of $R$ is less than or equal to the surface area of the square of the same circumference. Therefore,

$$
|P| \leqslant|R| \leqslant\left(\frac{a}{4}\right)^{2}
$$

We will also need the famous Pick Theorem [10].

Theorem 10 (Pick's Theorem). Let $P$ be a lattice polygon. Assume there are $i$ lattice points in the interior of $P$, and $b$ lattice points on its boundary. Then

$$
|P|=i+\frac{b}{2}-1 \text {. }
$$

Theorem 11. Let $G=P_{\infty} \square P_{\infty}$ and let $X \subset V(G)$ be finite. Then

$$
|X| \leqslant \frac{\left(\left|N_{G}(X)\right|-2\right)^{2}}{8}+\frac{1}{2} .
$$

Proof. We may assume that $G$ is in fact a plane graph (i.e. planar embedding of $G$ ) and that $X$ is non-empty. Let $S=N_{G}(X)$ and define $G_{S}$ as described above.

Suppose first that $X$ induces a connected subgraph in $G$. Then the edges belonging to the external (infinite) face of $G_{S}$ form a cycle - we denote it by $C$ and note that $|C| \leqslant\left|N_{G}(X)\right|$, where by $|C|$ we understand the number of edges (or equivalently vertices) of $C$. The region of the plane inside $C$ we denote by $F$; note it includes all vertices of $X$. Next we replace each edge $e$ of length 1 of $C$ with a polyline consisting of two line segments joining the ends of $e$ with the middle of the unit square incident to $e$ which lies outside $F$. Clearly the polyline has length $\sqrt{2}$. The edges of length $\sqrt{2}$ of $C$ remain unchanged. Note that by the definition of $G_{S}$, the middle of such a unit square may belong to at most one of those polylines of length $\sqrt{2}$. Thus this way we obtain a polygon $P$ with $c(P)=|C| \sqrt{2}$ and with angles $\pi / 2$ and $3 \pi / 2$ only, which contains $F$. By Proposition 9,

$$
|P| \leqslant\left(\frac{|C| \sqrt{2}}{4}\right)^{2}=\frac{|C|^{2}}{8} .
$$




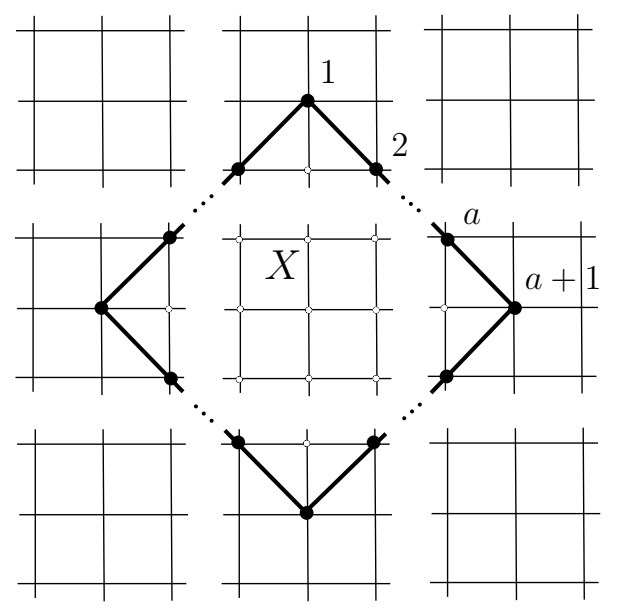

Figure 2: Illustrating the sharpness of Theorem 11 (black dots are elements of $N_{G}(X)$ ): $\left|N_{G}(X)\right|=4 a,|X|=2 \cdot \sum_{i=2}^{a}(2 i-3)+2 a-1=2 a^{2}-2 a+1=(4 a-2)^{2} / 8+1 / 2$.

Thus by Pick's theorem,

$$
\frac{|C|^{2}}{8} \geqslant|P| \geqslant|F| \geqslant|X|+|C| / 2-1
$$

and consequently,

$$
|X| \leqslant \frac{(|C|-2)^{2}}{8}+\frac{1}{2} \leqslant \frac{\left(\left|N_{G}(X)\right|-2\right)^{2}}{8}+\frac{1}{2}
$$

as $\left|N_{G}(X)\right| \geqslant|C| \geqslant 4$.

Suppose now that $G[X]$ has two components, and let $X_{1}, X_{2}$ induce these (hence $X=X_{1} \cup X_{2}$ ). If the edges belonging to the external (infinite) face of $G_{S}$ form a cycle, we proceed as above. Otherwise, $\left|\left(X_{1} \cup N_{G}\left(X_{1}\right)\right) \cap\left(X_{2} \cup N_{G}\left(X_{2}\right)\right)\right|=\left|N_{G}\left(X_{1}\right) \cap N_{G}\left(X_{2}\right)\right| \leqslant 1$, and thus we may shift one of the components (i.e., isomorphically map it to a subgraph of $G$ ) so that no vertex of $X_{1}$ and $X_{2}$ overlaps, but $\left|\left(X_{1} \cup N_{G}\left(X_{1}\right)\right) \cap\left(X_{2} \cup N_{G}\left(X_{2}\right)\right)\right| \geqslant 2$. Then the external neighbourhood of $X$ gets smaller, but the cardinality of $X$ does not change, while the edges belonging to the external (infinite) face of $G_{S}$ form a cycle now, and again the theorem follows by the reasoning above.

If finally $G[X]$ has more than two components, we proceed as in the previous paragraph, shifting components one by one, not changing the size of $X$, and potentially diminishing the cardinality of external neighbourhood of $X$, until the edges belonging to the external face of $G_{S}$ form a cycle, and then we conclude as above.

We note that the bound in Theorem 11 above cannot be improved in general, which is exemplified in the Figure 2 below (where $\left|N_{G}(X)\right|$ is divisible by 4 ). 


\subsection{Proof of Theorem 4}

Proof of Theorem 4. Let $C=C_{n} \square C_{n}$ with $n \geqslant 3$ be standardly embedded on the torus and suppose the thesis does not hold for a given $k \in\left[1, n^{2}-1\right]$. Consider any $S \subset V(C)$ with $|S|=k$ such that $m(G, S)=m(G, k)$, and let $X_{1}, \ldots, X_{q}$ be the vertex sets of the components of $C-S$, hence if only $2 n-1 \leqslant k<\frac{n^{2}}{2}$, then for every $i=1, \ldots, q$,

$$
\begin{aligned}
\left|X_{i}\right| & <\frac{n^{2}-(2 n-1)}{(2 n-1)^{2}} \cdot\left(n^{2}-(2 n-1)+n \sqrt{n^{2}-2(2 n-1)}\right) \\
& =\frac{(n-1)^{2} \cdot\left((n-1)^{2}+n \sqrt{n^{2}-4 n+2}\right)}{(2 n-1)^{2}} \\
& \leqslant \frac{(n-1)^{4}+(n-1)^{2} n(n-2)}{(2 n-1)^{2}} \\
& =\frac{2\left(n^{2}-2 n+1\right)\left(n^{2}-2 n+\frac{1}{2}\right)}{(2 n-1)^{2}} \\
& \leqslant \frac{2\left(n^{2}-2 n+\frac{3}{4}\right)^{2}}{(2 n-1)^{2}} \\
& =\frac{2\left[\left(n-\frac{1}{2}\right)\left(n-\frac{3}{2}\right)\right]^{2}}{(2 n-1)^{2}} \\
& =\frac{(2 n-3)^{2}}{8} .
\end{aligned}
$$

Any maximal connected surface obtained from the torus after removing all points of the edges and vertices of $C_{S}$ will be naturally called a face. Let $F_{i}$ be the face of $C_{S}$ containing $X_{i}$, for $i=1, \ldots, q$. Let further $F_{q+1}, \ldots, F_{p}$ be the remaining faces of $C_{S}$ - note that their interiors do not contain any vertex of $V(C) \backslash S$, and thus they can only be triangles or squares. Now for $i=1, \ldots, p$, denote by $B_{i}$ the boundary walk or walks of the face $F_{i}$, and let $\left|B_{i}\right|$ be the cardinality of the set of vertices of $B_{i}$. By $l\left(B_{i}\right)$ we in turn denote the length of $B_{i}$, that is the number of edges appearing in it where an edge appearing two times in the walk or walks (i.e. an edge incident with $F_{i}$ from both sides) is counted twice. Consequently, $l\left(B_{i}\right) \geqslant\left|B_{i}\right|,\left|B_{i}\right|=\left|N_{C}\left(X_{i}\right)\right|$ and $N_{C}\left(X_{i}\right) \subseteq S$, for $i=1, \ldots, q$.

For any fixed integer $i \in[1, n]$, the set of vertices $\{(i, j): j \in[1, n]\}$ will be called the $i$-th row, while the set $\{(j, i): j \in[1, n]\}$ will be called the $i$-th column of $C=C_{n} \square C_{n}$, cf. the standard notation discussed at the beginning of Section 3.1. Any set of vertices $X^{\prime} \subseteq V(C)$ will be called vast if it contains vertices from at least $n-1$ rows or at least $n-1$ columns of $C$, i.e. $X^{\prime}$ is vast if there are at least $n-1$ indices $i$ for which there exists an index $j_{i}$ with $\left(i, j_{i}\right)$ in $X^{\prime}$ or at least $n-1$ indices $i$ for which there exists an index $j_{i}$ with $\left(j_{i}, i\right)$ in $X^{\prime}$.

Claim 12. If $X^{\prime} \subseteq V(C)$ is not vast and $C\left[X^{\prime}\right]$ is connected, then

$$
\left|X^{\prime}\right| \leqslant \frac{\left(\left|N_{C}\left(X^{\prime}\right)\right|-2\right)^{2}}{8}+\frac{1}{2}
$$


Proof. We first note that $C\left[X^{\prime}\right]$ can be isomorphically mapped to a subgraph of $P_{\infty} \square P_{\infty}$ so that the external neighbourhoods of the both subgraphs have the same cardinality. To see this, note that since $X^{\prime}$ is connected and not vast, there must be two consecutive rows of $C$ and two consecutive columns of $C$ without elements of $X^{\prime}$, and thus if we delete from $C$ all edges between these two rows and all edges between these two columns, then the resulting $C^{\prime}$ will be planar and thus clearly isomorphic to a subgraph of $P_{\infty} \square P_{\infty}$, whereas $C\left[X^{\prime}\right]$ is also a subgraph of $C^{\prime}$ with the same external neighbourhood as in $C$. The claim thus follows by Theorem 11.

Claim 13. If $X^{\prime} \subseteq V(C)$ is not vast and $\left|N_{C}\left(X^{\prime}\right)\right| \leqslant 2 n-2$, then

$$
\left|X^{\prime}\right| \leqslant \frac{\left(\left|N_{C}\left(X^{\prime}\right)\right|-2\right)^{2}}{8}+\frac{1}{2} \text {. }
$$

Proof. We are going to proceed similarly as above. We might however need to introduce some modifications of $X^{\prime}$ beforehand. We first show that we may actually assume there are two consecutive rows of $C$ without elements of $X^{\prime}$.

Indeed, for suppose this is not the case. First, assume that row $i$, for some $i \in[1, n]$, does not intersect $N_{C}\left(X^{\prime}\right)$. Then, as $X^{\prime}$ is not vast, row $i$ contains no element in $X^{\prime}$ either. In addition, row $i-1(\bmod n)$ cannot contain an element in $X^{\prime}$ either, and hence we obtain two consecutive rows not intersecting $X^{\prime}$, a contradiction. We therefore may assume that every row intersects $N_{C}\left(X^{\prime}\right)$. As $\left|N_{C}\left(X^{\prime}\right)\right| \leqslant 2 n-2$, this implies that there are (at least) two rows each containing exactly one element of $N_{C}\left(X^{\prime}\right)$. These two rows thus contain no element of $X^{\prime}$, as $X^{\prime}$ is not vast, and due to our supposition these cannot be two consecutive rows in $C$. Assume thus without loss of generality that these are rows 1 and $r \in[3, n-1]$. We then modify $X^{\prime}$ by exchanging each element $(a, b) \in X^{\prime}$ with $a \in[2, r]$ by $(a-1, b)$. This way, neither the size of $X^{\prime}$ nor the cardinality of its external neighbourhood will change. Moreover $X^{\prime}$ will still not be vast and there will be two consecutive rows $(r-1$ and $r)$ without elements of $X^{\prime}$.

The above potential changes do not modify the columns of the elements in $X^{\prime}$, and therefore we may also assume, by symmetry, that there are two consecutive columns, say $c-1$ and $c$ in $C$ without elements of $X^{\prime}$. We therefore may delete the edges of $C$ between rows of $r-1$ and $r$ and between the columns $c-1$ and $c$, and the thesis follows by Theorem 11 via the same reasoning as in the proof of Claim 12 above.

Claim 14. If $X^{\prime} \subseteq V(C)$ is vast and $\left|N_{C}\left(X^{\prime}\right)\right| \leqslant 2 n-2$ then $X^{\prime}$ contains at least $n-1$ elements from some row and at least $n-1$ elements from some column of $C$.

Proof. Without loss of generality, suppose $X^{\prime}$ contains at least one vertex in rows $1, \ldots, n-$ 1. Suppose that $X^{\prime}$ contains at most $n-2$ elements from each of the rows of $C$. Then however $N_{C}\left(X^{\prime}\right)$ must include at least 2 elements from each of the rows $1, \ldots, n-1$, and also from row $n$ if some element from this row belongs to $X^{\prime}$, or otherwise $N_{C}\left(X^{\prime}\right)$ includes at least one element from this row. In both cases we obtain a contradiction with the assumption that $\left|N_{C}\left(X^{\prime}\right)\right| \leqslant 2 n-2$, and hence $X^{\prime}$ contains at least $n-1$ elements from some row of $C$. But it means $X^{\prime}$ contains at least one vertex in at least $n-1$ columns 


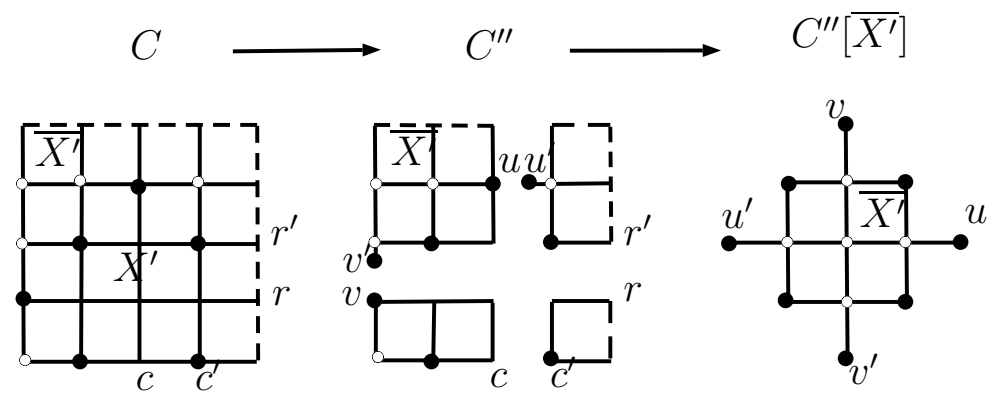

Figure 3: Exemplary construction of $C^{\prime \prime}$ and embedding in $P_{\infty} \square P_{\infty}$.

of $C$ and by a symmetrical reasoning as above we conclude $X^{\prime}$ must also contain at least $n-1$ elements from some column of $C$.

For any $X^{\prime} \subseteq V(C)$, denote $\overline{X^{\prime}}:=V(C) \backslash\left(X^{\prime} \cup N_{C}\left(X^{\prime}\right)\right)$, and note that $N_{C}\left(\overline{X^{\prime}}\right) \subseteq N_{C}\left(X^{\prime}\right)$. Claim 15. If $X^{\prime} \subseteq V(C)$ is vast and $\left|N_{C}\left(X^{\prime}\right)\right| \leqslant 2 n-3$ then $\overline{X^{\prime}}$ is not vast.

Proof. Suppose the claim is false, i.e. that $\overline{X^{\prime}}$ is vast. As $N_{C}\left(\overline{X^{\prime}}\right) \subseteq N_{C}\left(X^{\prime}\right)$, by Claim 14, $X^{\prime}$ contains at least $n-1$ elements from some row, while $\overline{X^{\prime}}$ contains at least $n-1$ elements from another row of $C$. This implies there has to be an element of $N_{C}\left(X^{\prime}\right)$ in each column of $C$, and moreover there have to be at least two such elements in at least $n-2$ columns. Therefore we obtain a contradiction, as $\left|N_{C}\left(X^{\prime}\right)\right|<2 n-2$.

Claim 16. If $X^{\prime} \subseteq V(C)$ is vast, $\left|N_{C}\left(X^{\prime}\right)\right|=2 n-2$ and $\overline{X^{\prime}}$ is vast, then

$$
\left|X^{\prime}\right| \geqslant \frac{(n-1)^{2}}{2}+\frac{1}{2}
$$

Proof. We will first embed $\overline{X^{\prime}}$ into a subgraph of $P_{\infty} \square P_{\infty}$ whose external neighbourhood has at most 2 more vertices than that of $\overline{X^{\prime}}$ in $C$.

Since $N_{C}\left(\overline{X^{\prime}}\right) \subseteq N_{C}\left(X^{\prime}\right)$, then $\left|N_{C}\left(\overline{X^{\prime}}\right)\right| \leqslant 2 n-2$. Thus, by Claim 14, $\overline{X^{\prime}}$ contains at least $n-1$ elements from some row and at least $n-1$ elements from some column of $C$. Similarly, $X^{\prime}$ contains at least $n-1$ elements from some row $r$ and at least $n-1$ elements from some column $c$ of $C$. Thus, each of these two rows and two columns must contain exactly $n-1$ elements from the respective sets (as otherwise there would be an edge between $X^{\prime}$ and $\overline{X^{\prime}}$ ). Then we create a graph $C^{\prime}$ of $C$ by deleting all edges between column $c$ and its neighbouring column $c^{\prime}$ (arbitrarily chosen out of the two) and all edges between row $r$ and its neighbouring row $r^{\prime}$. Note that as all the removed edges, except possibly two are incident with vertices in $X^{\prime}$, these cannot be incident with $\overline{X^{\prime}}$. We thus might have deleted at most two edges incident with vertices in $\overline{X^{\prime}}$, and for these we provide substitutes in the following manner. If $u$ is a (single) vertex $u$ from column $c$ which belongs to $N_{C}\left(\overline{X^{\prime}}\right)$, we introduce a new vertex $u^{\prime}$ and join it by an edge with the neighbour (in $C$ ) of $u$ in column $c^{\prime}$. Analogously, if $v$ is a (single) vertex $v$ from 


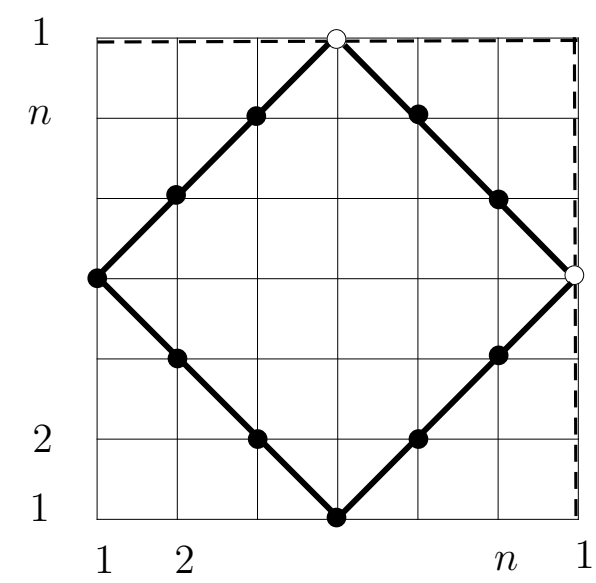

Figure 4: Illustrating sharpness in Theorem 4 for $k=2 n-2$

(black dots are elements of $S$ ).

row $r$ which belongs to $N_{C}\left(\overline{X^{\prime}}\right)$, then we introduce a new vertex $v^{\prime}$ and join it by an edge with the neighbour (in $C$ ) of $v$ in row $r^{\prime}$. The resulting graph (with at most two new vertices) we denote by $C^{\prime \prime}$, cf. the example in Figure 3. Analogously as previously, $C^{\prime \prime}\left[\overline{X^{\prime}}\right]$ (and thus also $C\left[\overline{X^{\prime}}\right]$ ) is isomorphic with a subgraph of $P_{\infty} \square P_{\infty}$ whose external neighbourhood in $P_{\infty} \square P_{\infty}$ contains at most $\left|N_{C}\left(\overline{X^{\prime}}\right)\right|+2 \leqslant\left|N_{C}\left(X^{\prime}\right)\right|+2=2 n$ elements. Thus by Theorem 11,

$$
\left|\overline{X^{\prime}}\right| \leqslant \frac{(2 n-2)^{2}}{8}+\frac{1}{2}=\frac{(n-1)^{2}+1}{2}
$$

and hence

$$
\left|X^{\prime}\right| \geqslant n^{2}-(2 n-2)-\frac{(n-1)^{2}+1}{2}=\frac{(n-1)^{2}}{2}+\frac{1}{2} .
$$

Before we show how one may derive Theorem 4 from the presented claims, we note that the bound from Claim 16 above (similarly as the one in Claim 12) is sharp, which is exemplified in Figure 4.

Assume first that $k \leqslant 2 n-2$. We note that then at least one $X_{i}$ is vast. Indeed, if it were otherwise, each row (and column) of $C$ would have to contain at least two elements of $S$, hence $|S| \geqslant 2 n$, a contradiction. Without loss of generality assume $X_{1}$ is vast.

Now denote $X_{0}:=X_{2} \cup X_{3} \cup \ldots \cup X_{q}$ and suppose that $X_{0}$ is not vast (in particular we may have $\left.X_{0}=\emptyset\right)$. Since $\left|N_{C}\left(X_{0}\right)\right| \leqslant|S| \leqslant 2 n-2$, by Claim 13 ,

$$
\left|X_{0}\right| \leqslant \frac{(k-2)^{2}}{8}+\frac{1}{2}
$$

hence,

$$
\left|X_{1}\right| \geqslant n^{2}-k-\left(\frac{(k-2)^{2}}{8}+\frac{1}{2}\right)=n^{2}-\frac{(k+2)^{2}}{8}-\frac{1}{2}
$$


and we obtain a contradiction (thus the thesis of Theorem 4 follows).

So we may assume that $X_{0}$ is vast, hence $\overline{X_{1}}$ is vast as well (as $X_{0} \subseteq \overline{X_{1}}$ ). By Claim 15 this implies that $k=2 n-2$. We will show that then we must have $q<3$. By Claim 14 each of $X_{1}$ and $X_{0}$ contains at least $n-1$ vertices from some row of $C$, hence none of these contains all elements from any column of $C$, and thus each column contains a vertex from $S$. Moreover, as any $n-1$ vertices of $X_{0}$ from the same row induce a connected subgraph of $C$, and hence they must belong to the same $X_{i}$, we may assume without loss of generality that there is such a set of vertices in $X_{2}$. Observe that if any given column $c$ intersects $i$ different sets among $X_{1}, \ldots, X_{q}$, then $c$ contains at least $i$ elements of $S$. Therefore, as each of $X_{1}$ and $X_{2}$ contains $n-1$ elements of some row, at least $n-2$ columns must contain at least 2 elements in $S$, summing to no less than $2 n-4$ elements of $S$. Since the remaining two columns contain at least 2 elements in $X_{1} \cup X_{2}$, these imply the existence of at least $(2 n-4)+2=2 n-2=|S|$ elements in $S$. Thus indeed $q<3$, as any element $v$ in $X_{j}$ with $j>2$ would imply the existence of an element in $S$ not yet counted in the column of $v$. Since $q \leqslant 2$, we must however have

$$
\max _{1 \leqslant i \leqslant q}\left|X_{i}\right| \geqslant \frac{1}{2}\left(n^{2}-k\right)=\frac{(n-1)^{2}}{2}+\frac{1}{2}
$$

hence we obtain a contradiction (thus the thesis of Theorem 4 follows).

As the case when $\frac{n^{2}}{2} \leqslant k<n^{2}$ is trivial, we may assume till the end of the proof that $2 n-1 \leqslant k<\frac{n^{2}}{2}$. We will show that then for every $i=1, \ldots, q$,

$$
\left|X_{i}\right| \leqslant \frac{\left(\left|B_{i}\right|-2\right)^{2}}{8}+\frac{1}{2}
$$

This follows by Claim 12 when $X_{i}$ is not vast. So suppose now that $X_{i}$ is vast. In the case when $\left|N_{C}\left(X_{i}\right)\right| \leqslant 2 n-3$ (hence $\overline{X_{i}}$ is not vast by Claim 15) and in the case when $\left|N_{C}\left(X_{i}\right)\right|=2 n-2$ and $\overline{X_{i}}$ is not vast, by Claim 13 ,

$$
\left|\overline{X_{i}}\right| \leqslant \frac{(2 n-4)^{2}}{8}+\frac{1}{2}
$$

and hence,

$$
\begin{aligned}
\left|X_{i}\right| & \geqslant n^{2}-(2 n-2)-\left(\frac{(2 n-4)^{2}}{8}+\frac{1}{2}\right)>(n-1)^{2}-\frac{(n-2)^{2}}{2} \\
& >\frac{(n-1)^{2}}{2}>\frac{(2 n-3)^{2}}{8},
\end{aligned}
$$

a contradiction with (3). Similarly, in the case when $\left|N_{C}\left(X_{i}\right)\right|=2 n-2$ and $\overline{X_{i}}$ is vast, we obtain a contradiction with (3) by Claim 16. Thus we may assume that $\left|N_{C}\left(X_{i}\right)\right| \geqslant 2 n-1$. Then however, by (3),

$$
\left|X_{i}\right|<\frac{(2 n-3)^{2}}{8}<\frac{\left(\left|B_{i}\right|-2\right)^{2}}{8}+\frac{1}{2}
$$


and thus (4) follows.

By Euler's formula for graphs embeddable without crossings on the torus:

$$
2 k+2 p \geqslant 2\left|E\left(G_{S}\right)\right|=\sum_{i=1}^{p} l\left(B_{i}\right) \geqslant \sum_{i=1}^{p}\left|B_{i}\right| .
$$

Thus, for $x:=\max _{1 \leqslant i \leqslant q}\left|X_{i}\right|$, by (4),

$$
\begin{aligned}
2 k & \geqslant \sum_{i=1}^{p}\left(\left|B_{i}\right|-2\right) \geqslant \sum_{i=1}^{q}\left(\left|B_{i}\right|-2\right)=\sum_{i=1}^{q} \sum_{v \in X_{i}} \frac{\left|B_{i}\right|-2}{\left|X_{i}\right|} \\
& \geqslant \sum_{i=1}^{q} \sum_{v \in X_{i}} \frac{\sqrt{8\left|X_{i}\right|-4}}{\left|X_{i}\right|} \geqslant \sum_{i=1}^{q} \sum_{v \in X_{i}} \frac{\sqrt{8 x-4}}{x} \\
& =\frac{\sqrt{8 x-4}}{x} \sum_{i=1}^{q} \sum_{v \in X_{i}} 1=\frac{\sqrt{8 x-4}}{x}\left(n^{2}-k\right) .
\end{aligned}
$$

Therefore, taking into account the fact that $x \geqslant 1\left(\right.$ for $k<n^{2}$ ),

$$
x \geqslant \frac{x}{2-\frac{1}{x}} \geqslant\left(\frac{n^{2}-k}{k}\right)^{2}
$$

and

$$
\left(\frac{k}{n^{2}-k}\right)^{2} x^{2}-2 x+1 \geqslant 0,
$$

where the polynomial in $x$ on the left-hand side of the inequality above has two roots (for $\left.k<\frac{n^{2}}{2}\right)$ :

$$
x_{1}=\left(\frac{n^{2}-k}{k}\right)^{2}\left(1-\sqrt{1-\left(\frac{k}{n^{2}-k}\right)^{2}}\right)<\left(\frac{n^{2}-k}{k}\right)^{2}
$$

and

$$
x_{2}=\left(\frac{n^{2}-k}{k}\right)^{2}\left(1+\sqrt{1-\left(\frac{k}{n^{2}-k}\right)^{2}}\right)>\left(\frac{n^{2}-k}{k}\right)^{2},
$$

hence, by (6),

$$
x \geqslant x_{2}=\frac{n^{2}-k}{k^{2}} \cdot\left(n^{2}-k+n \sqrt{n^{2}-2 k}\right),
$$

a contradiction. 


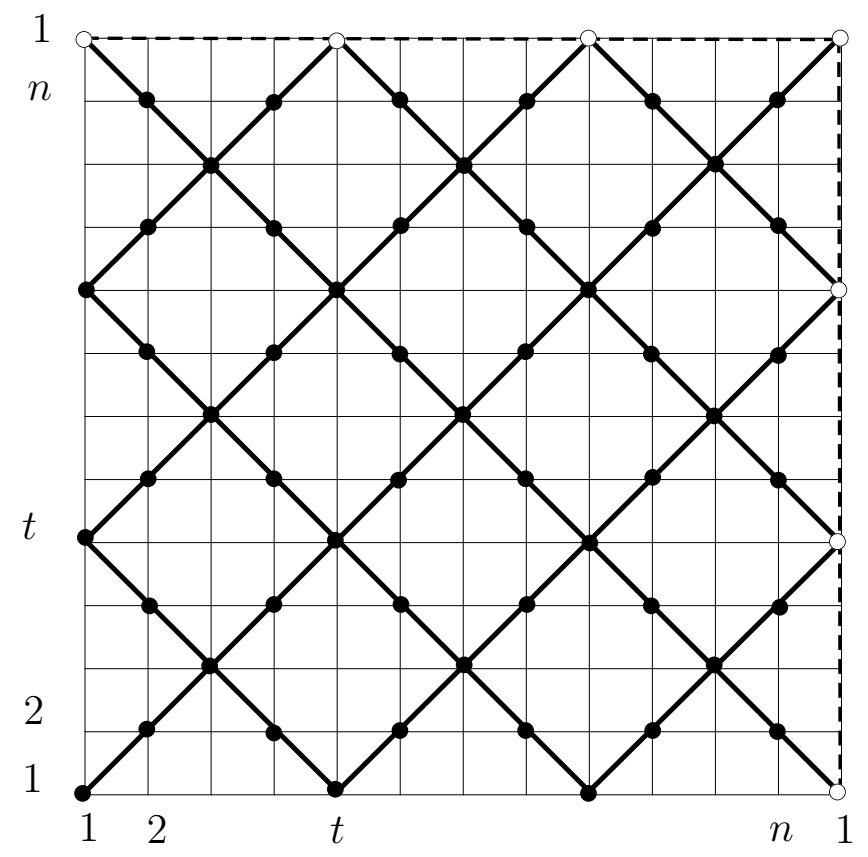

Figure 5: Illustrating the sharpness in Theorem 4 for $2 n-1 \leqslant k<n^{2} / 2$

(black dots are elements of $S$ ).

\subsection{Sharpness}

The bound in Theorem 4 is best possible in infinitely many cases. Let e.g. $n=s(t-1)$ for some $s \geqslant 1, t \geqslant 2$, and consider the set $S$ as displayed in Figure 5 below.

Each face $F_{i}$ is a square of diagonal $t-1$. Thus the side of each $F_{i}$ is of length $\frac{t-1}{\sqrt{2}}$. Hence the area and the circumference of $F_{i}$ are equal to

$$
\begin{aligned}
\left|F_{i}\right| & =\frac{(t-1)^{2}}{2} \\
c\left(F_{i}\right) & =4 \frac{t-1}{\sqrt{2}} .
\end{aligned}
$$

By (7), the number $p$ of faces satisfies

$$
p=n^{2} \div \frac{(t-1)^{2}}{2}=2 s^{2}
$$

Since each edge lying on a boundary of every face has length $\sqrt{2}$, by (8), the boundary of each face has $2(t-1)$ edges. Thus the total number of edges is equal to

$$
|E|=\frac{1}{2} \cdot 2 s^{2} \cdot 2(t-1)=2 s^{2}(t-1) .
$$

Therefore, by Euler's formula

$$
k=|V|=|E|-|F|=2 s^{2}(t-2) .
$$


By (7) and Pick's theorem

$$
\left|X_{i}\right|=\frac{(t-1)^{2}}{2}-(t-1)+1=\frac{t^{2}-4 t+5}{2} .
$$

Finally the lower bound in Theorem 4 is

$$
\begin{aligned}
\left|X_{i}\right| \geqslant & \frac{\left(n^{2}-k\right)\left(\left(n^{2}-k\right)+n \sqrt{n^{2}-2 k}\right)}{k^{2}} \\
& =\frac{s^{2}\left(t^{2}-4 t+5\right)\left(s^{2}\left(t^{2}-4 t+5\right)+s(t-1) \sqrt{s^{2}(t-1)^{2}-4 s^{2}(t-2)}\right)}{4 s^{4}(t-2)^{2}} \\
& =\frac{s^{2}\left(t^{2}-4 t+5\right)\left(s^{2}\left(t^{2}-4 t+5\right)+s(t-1) s(t-3)\right)}{4 s^{4}(t-2)^{2}} \\
& =\frac{s^{2}\left(t^{2}-4 t+5\right)\left(s^{2}\left(t^{2}-4 t+5\right)+s^{2}\left(t^{2}-4 t+3\right)\right)}{4 s^{4}(t-2)^{2}} \\
& =\frac{\left(t^{2}-4 t+5\right)\left(2 t^{2}-8 t+8\right)}{4(t-2)^{2}}=\frac{t^{2}-4 t+5}{2},
\end{aligned}
$$

which agrees with (12).

The example discussed above shows that the result of Theorem 4 cannot be improved in general for the cases when $k \geqslant 2 n-1$. A representative of an infinite family of examples for sharpness of the bound in Theorem 4 when $k=2 n-2$ is displayed in Figure 4, while Figure 2 indicates how one can construct such examples in the remaining case, i.e. when $k$ is small, that is $k \leqslant 2 n-3$.

\section{References}

[1] Ajtai M., Alon N., Bruck J., Cypher R., Ho C.T., Naor M. and Szemerédi E., Fault tolerant graphs, perfect hash functions and disjoint paths, in Proc. IEEE Symp. on Foundations of Computer Science, pages 693-702, 1992.

[2] Alon N., Chung F., Explicit construction of linear sized tolerant networks, Discrete Math., 72:15-19, 1988.

[3] Bagga, K.S., L.W. Beineke, W.D. Goddard, M.J. Lipman and R.E. Pippert, A survey of integrity, Discrete Appl. Math., 37/38:13-28, 1992.

[4] Balogh J., Mészáros T., Wagner A.Z., Two results about the hypercube, Disrete Appl. Math., 247:322-326, 2018.

[5] L. W. Beineke, W. Goddard, P. Hamburger, D. J. Kleitman, M. J. Lipman and R. E. Pippert, The integrity of the cube is small, J. Combin. Math. Combin. Comput., 9:191-193, 1991.

[6] Benko D., Ernst C., Lanphier D., Asymptotic bounds on the integrity of graphs and separator theorems for graphs, SIAM J. Discrete Math., 23:265-277, 2009. 
[7] Dudek A., Szymański A., Zwonek M., $(H ; k)$-stable graphs with minimum size, Discuss. Math. Graph Theory, 28:137-149, 2008.

[8] Erdős P., Graham R.L., Szemerédi E., On sparse graphs with dense long paths, Comp. Math. Appl., 1:365-369, 1975.

[9] Hayes J.P., A graph model for fault-tolerant computing systems, IEEE Trans. Comput., 25(9):875-884, 1976.

[10] Pick G., Geometrisches zur Zahlenlehre, Sitzungsberichte Lotos (Prag), Natur-med. Verein für Böhmen, 19:311-319, 1899.

[11] Ueno S., Bagchi A., Hakimi S.L., Schmeichel E.F., On Minimum Fault-Tolerant Networks, SIAM J. Discrete Math., 6(4): 565-574, 1993.

[12] Ueno S., Yamada T., Optimal fault-tolerant linear arrays, in Proceedings of the Fifteenth Annual ACM Symposium on Parallel Algorithms and Architectures, pages 60-64, 2003.

[13] Vince A., The integrity of a cubic graph, Discrete Appl. Math., 140:223-239, 2004.

[14] Zhang L., Fault tolerant networks with small degree, in Proceedings of the Twelfth Annual ACM Symposium on Parallel Algorithms and Architectures, pages 64-69, 2000.

[15] A. Żak, General lower bound on the size of $(H ; k)$-stable graphs, J. Comb. Optim. 29(2):367-372, 2015. 